hybridized in the presence of Cy5-labelled targets derived from the actin-related genes. For the arrays and protocols used here, most actin-specific 25-mer probes did not hybridize to the actin-related targets. Those probes that showed detectable cross-hybridization were readily predicted using BLAST analysis. Crosshybridizing probes could also be predicted with reasonable accuracy by including probes complementary to homologous targets on the experimental array. Low ratios of cross-hybridization control probe signal-to-target-specific probe signal were indicative of lower levels of cross-hybridization. This approach may offer a general method for experimentally verifying probe specificity.

Sherman, Fred

\section{DNA microarray analysis of strains lack- ing BTN1, the yeast ortholog of the human Batten disease gene}

\section{David A. Pearce, Tracy Ferea, Seth A. Nosel, Biswadip Das \& Fred Sherman}

University of Rochester M edical School, Department of Biochemistry and Biophysics, Rochester, New York 14642, USA

Batten disease is the most common form of Neuronal ceroid-lipofuscinoses (NCL), the most frequently occurring group of progressive neurodegenerative diseases in children with an incidence as high as one in 12,500 live births, and with about 440,000 carriers in the USA. These disorders are autosomal recessive with progression characterised by visual problems leading to blindness, decline in mental abilities, increased severity of untreatable seizures, blindness, loss of motor skills and premature death. Although the gene responsible for Batten disease, CLN3, was positionally cloned in 1995 , the function of $\operatorname{Cln} 3 p$ is still unknown. We previously reported that the yeast Saccharomyces cerevisiae contains a homolog to $C \ln 3 p$, designated Btn $1 p$, and that the human Cln $3 p$ complemented the pH-dependent resistance to D-(-)-threo-2-amino-1-[p-nitrophenyl]1,3-propanediol in btn1-D yeast mutants. Furthermore, the severity of Batten disease in humans and the degree of ANP resistance in yeast are related when the equivalent changes in $C \ln 3 p$ and Btn1p are compared. This establishes that the yeast and human protein perform the same function in the cell.

Examination of the expression of virtually all yeast genes by DNA microarray analysis of $B T N 1$ and $b t n 1$-D strains revealed differential expression of two genes, HSP30 and BTN2 (YGR142W). Expression of both HSP30, a down regulator of plasma membrane ATPase, and BTN2 is increased in btn1-D strains.

Our biochemical studies have revealed that the $\mathrm{pH}$-dependent resistance to ANP of btn 1-D strains results from an elevated ability to acidify the growth medium. This increased rate of acidification is due to an increased activity of the plasma membrane ATPase, during the early phases of growth. Yeast lacking Btn $1 \mathrm{p}$ have a vacuolar $\mathrm{pH}$ of 5.8 , as compared to normal, 6.2, in the early phases of growth. As btn 1 -D strains grow, the elevated plasma membrane ATPase activity and the decrease in vacuolar $\mathrm{pH}$ are apparently normalised. A link between vacuolar $\mathrm{pH}$ and plasma membrane ATPase activity was confirmed in strains which

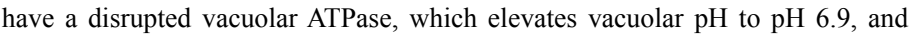
results in a lower than normal plasma membrane ATPase activity. We propose that btn1-D strains have increased expression of HSP30 in response to an elevated plasma membrane ATPase activity due to an altered regulation of vacuolar $\mathrm{pH}$. The increased expression of $B T N 2$, a previously unknown gene that has homology to human $H O O K 1$, whose Drosophila homolog is a novel endocytic component may suggest a role for this protein in balancing intracellular $\mathrm{pH}$.
Shroff, Robert

\section{Genome-wide mapping of meiosis- induced DNA double-strand breaks}

\author{
Robert Shroff \& M ichael Lichten
}

Laboratory of Biochemistry, DBS, NCI, NIH, Bethesda, M aryland 20892, USA

The major applications of DNA microarray technology to date have been genomewide analysis of gene expression and analysis of DNA variation. Our goal is to develop an approach using DNA microarray technology to quantitatively study the distribution of DNA-associated proteins at the whole-genome level. An initial approach will determine the genomic distribution of DNA double-strand breaks (DSBs) that initiate meiotic recombination in Saccharomyces cerevisiae. DSB formation involves an intermediate stage where DNA ends are covalently linked to Spo11p. In a rad50S mutant background these intermediates accumulate throughout meiosis. Our approach is to purify Spo11p::His/DNA intermediates by immobilized metal-affinity chromatography. The DNA purified from these intermediates is then hybridized to DNA microarrays to map the distribution and frequency of Spo11p-linked double-strand breaks throughout the genome. With the addition of a formaldehyde cross-linking step, this approach could be used to map the distribution of any protein that interacts with the genome, either independently or as part of a protein complex. The affinity chromatography approach allows large amounts of material to be purified, and should enable the detection of minor or transient protein/DNA intermediates.

Sinibaldi, Ralph

\section{OpArraysm: covalently-bound DNA microarrays}

\section{Ralph M. Sinibaldi, M ike Chen, Ken Beckman, Sepehr Saljoughi, John ten Bosch, Nico Tuason, Chris Seidel, Sajeev Batra $\&$ Bob Saul}

O peron Technologies, 1000 Atlantic Ave., Alameda, California 94501, USA

We have developed a procedure of covalently attaching nucleic acids to 1"X 3" glass microscope slides. When we calculate the degree of target wash-off during hybridisation and subsequent washes (using oligonucleotides of $35 \mathrm{nt}$ to $45 \mathrm{nt}$ in length), we demonstrate a very stable attachment of the target nucleic acid to the glass slide. We measured the target wash-off using our chemistry and compared it to target wash-off experiments with published poly-L-lysine, aldehyde, UV crosslinking and amino chemistries. Our chemistry exhibits almost no wash-off whereas the other chemistries can exhibit as high as a $70 \%$ wash-off. We also wished to investigate different types of target nucleic acids on microarrays. Using our developed chemistry, we chose 96 yeast genes regulated during auxotrophic shift and designed and made three different types of target nucleic acids to be attached to microarrays. We made full-length cDNAs (500nt-2000nt), optimised amplified gene segments (400nt) and specific oligonucleotides (35nt-45nt) for comparison. These microarrays were hybridised to fluorescently-labeled cDNA made from yeast mRNA and the hybridisation signals were determined using a laser scanner. These experiments are designed to determine the relative sensitivity and reproducibility of these types of nucleic acid targets in gene expression experiments. The results of these experiments will be discussed. 